\title{
Androgen Receptor Signalling in Prostate Cancer: The Functional Consequences of Acetylation
}

\author{
Derek N. Lavery and Charlotte L. Bevan \\ Androgen Signalling Laboratory, Division of Cancer, Department of Surgery and Cancer, Imperial College London, \\ London W12 ONN, UK \\ Correspondence should be addressed to Derek N. Lavery, d.lavery@imperial.ac.uk
}

Received 11 August 2010; Accepted 16 November 2010

Academic Editor: Patrick Matthias

Copyright (C) 2011 D. N. Lavery and C. L. Bevan. This is an open access article distributed under the Creative Commons Attribution License, which permits unrestricted use, distribution, and reproduction in any medium, provided the original work is properly cited.

The androgen receptor (AR) is a ligand activated transcription factor and member of the steroid hormone receptor (SHR) subfamily of nuclear receptors. In the early stages of prostate carcinogenesis, tumour growth is dependent on androgens, and AR directly mediates these effects by modulating gene expression. During transcriptional regulation, the AR recruits numerous cofactors with acetylation-modifying enzymatic activity, the best studied include p300/CBP and the p160/SRC family of coactivators. It is known that recruitment of histone acetyltransferases (HATs) and histone deacetylases (HDACs) is key in finetuning responses to androgens and is thus likely to play a role in prostate cancer progression. Further, these proteins can also modify the AR itself. The functional consequences of AR acetylation, the role of modifying enzymes in relation to AR transcriptional response, and prostate cancer will be discussed.

\section{Introduction}

The androgen receptor (AR), a ligand-activated transcription factor and member of the steroid hormone subfamily of the nuclear receptor (NR) superfamily, mediates androgen signalling in the cell. The AR is the only NR that is coded on the $\mathrm{X}$-chromosome, thus males carrying a disease-associated mutation in the gene will be hemizygous and express the disease phenotype. The most prevalent AR-associated disease is prostate cancer, the fifth most commonly diagnosed cancer in the world and second most common cancer among men [1]. The prostate is a gland situated below the bladder, surrounding the urethra. The main function of the prostate is the secretion of components of the seminal fluid, hence, it plays a role in male fertility [2]. Prostate growth is dependent upon androgens, primarily testosterone and its more potent and physiologically active metabolite dihydrotestosterone $[3,4]$.

The AR is around 919 amino acids long and has distinct structural and functional domains [3, 5] (Figure 1). The $\mathrm{N}$-terminal domain of the receptor is highly flexible, with minimal secondary structure that upon protein-protein interactions can become more structured [6]. The central DNA-binding domain (DBD) is arranged into three alpha helices organised into two separate zinc finger-like motifs co-ordinated by eight cysteine residues. These helices are important in the recognition of specific DNA sequences, termed androgen response elements (AREs), and DNAdependent dimerisation of the receptor $[3,7]$. The ligandbinding domain (LBD) is situated in the AR C-terminal domain, is important in recognition and docking of androgens, and has been characterised by crystallography [8, 9]. Two transactivation domains exist in the AR: activation function-1 (AF1), situated in the N-terminal domain, and AF2, which is located in the LBD. Unlike other SHRs, and the majority of NRs, the main transactivation potential lies not within the LBD but within the $\mathrm{N}$-terminal domain of the AR $[6,10-12]$.

The molecular events leading to AR-regulated transcription are outlined in Figure 2. In the absence of androgens, the AR is located in the cytoplasm, in a complex with heat-shock and heat-shock-related proteins. Upon diffusion of androgen into the cell, the globular C-terminal domain of the receptor accepts ligand, and subsequent structural 


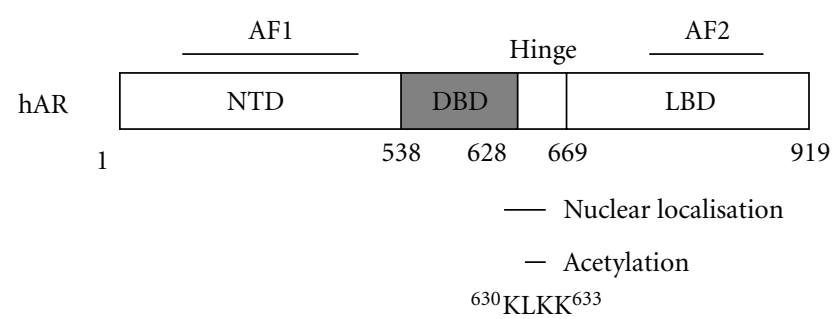

Figure 1: Schematic representation of the human androgen receptor (AR). The N-terminal domain (NTD) contains activation function-1 (AF1), which is the major region important in transcriptional activation. The central DNA-binding domain (DBD) is coordinated by two zinc finger motifs and recognises specific androgen response elements. The ligand-binding domain (LBD), situated in the C-terminus, is structurally well characterised and contains the ligand-dependent transactivation domain, AF2. The flexible hinge region connects the structured DBD and LBD and contains both a nuclear localisation sequence and acetylation motif.

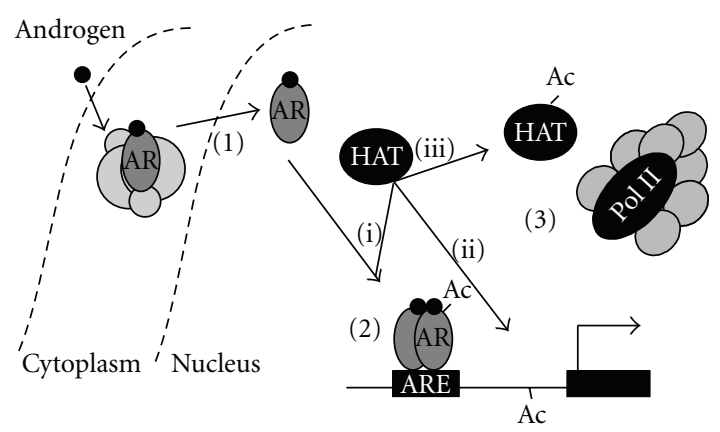

FIGURE 2: Role of acetylation in AR-regulated transcription. In general terms, ligand-dependent transcriptional activation by the AR can be described as follows: (1) ligand-bound AR dissociates from cytoplasmic heat-shock complexes and enters the nucleus. (2) AR binds to specific DNA sequences termed androgen response elements (AREs) and recruits members of the basal transcriptional apparatus. (3) AR recruits a variety of coregulators, which may serve to provide "platforms" for additional coregulator recruitment, regulate the architecture of chromatin directly, and ultimately intensify transcription from target genes. HATs function at a variety of stages including (i) direct acetylation of AR, (ii) acetylation of chromatin, and (iii) acetylation of other factors involved in transcriptional regulation. See text for details.

rearrangements result in dissociation of heat-shock proteins and the exposure of a nuclear localisation sequence (NLS) situated in the hinge region [3]. Nuclear AR binds to AREs located throughout the genome and recruits a variety of cofactors including members of the basal transcription complex and proteins with enzymatic activities such as HATs and HDACs. The formation and identities of these diverse multiprotein complexes result in tight transcriptional regulation of a variety of genes involved in prostate growth, maintenance, and differentiation $[6,13,14]$.

In the following review, the impact of acetylation on AR signalling will be discussed. First, modulation of AR activity by direct acetylation will be examined followed by the role of coregulator proteins with HAT/HDAC activity in
AR transcriptional complexes. Finally, the role of acetylation in prostate cancer formation/progression and the application of therapies will be discussed.

\section{Androgen Receptor Acetylation}

The AR polypeptide has a well-defined structure/function organisation. Increased sophistication and application of bioinformatic tools, the ability to produce high-quality recombinant proteins, and generation of modificationspecific monoclonal antibodies have aided the identification of multiple AR modifications. It is well known that the $\mathrm{AR}$ exists as a phosphoprotein, and its function is tightly regulated by residue-specific modification by a variety of kinases [15], but only relatively recently has a link been established between direct AR acetylation and protein function. In 2000, the Pestell laboratory identified a short motif $\left({ }^{630} \mathrm{KLKK}^{633}\right)$ within the hinge region of $\mathrm{AR}$ that has characteristics of an acetylation motif (RXKK) and is conserved between mouse, rat, and human AR (Figure 1) [16]. Using ${ }^{14} \mathrm{C}$-labelled acetate and recombinant GST-AR fragments, they were able to show that AR was acetylated by p300 and p300/cAMP-response element-binding proteinassociated factor (P/CAF) in vitro, and further immunoprecipitation experiments confirmed that AR was acetylated by these factors in vivo [16]. Combining a peptide-based approach with mass spectrometry, the group mapped the region of acetylation to the AR hinge region (amino acids 623-640) and specifically the KLKK motif and showed that mutation of this motif resulted in a drastically attenuated response to hormone. Furthermore, p300 and P/CAF were unable to potentiate mutant AR activity to the same degree as wild-type, suggesting that acetylation is required for maximal activation [16].

Elegant studies by the same laboratory using acetylationmimic/gain-of-function mutants confirmed that acetylation plays a key role in AR transactivation with functional consequences; such mutants interacted to a greater extent with p300 than wild-type receptor and enhanced prostate cancer cell growth on soft agar and in xenografts [17, 18]. Interestingly, $A R$ acetylation mimics showed reduced interactions with proteins with histone deacetylase activity (HDAC1) and also transcriptional corepressors (NCoR (Nuclear receptor CoRepressor), Smad3). It is known that p300 can act as a molecular scaffold, recruiting additional coactivators that may modulate the transcriptional response. As acetylation-mimic mutants interacted with p300 to a greater extent than wild-type AR, the authors performed additional cotransfection experiments to test a panel of coactivators. Indeed, it was found that these coactivators could activate acetylation mimics to a greater extent, presumably via increased $\mathrm{p} 300 / \mathrm{AR}$ interaction [17].

In addition to $\mathrm{p} 300$ and $\mathrm{P} / \mathrm{CAF}$, a third protein has been identified that directly acetylates AR, termed Tat-interactive protein, $60 \mathrm{kDa}$ (TIP60) [19]. Originally identified in a yeast two-hybrid screen using domain fragments of AR as bait, TIP60 was shown to interact with the AR-LBD [20]. In this assay, no interaction was observed between TIP60 and 
AR N-terminal and DNA-binding domains suggesting that TIP60 may be involved in ligand-dependent transactivation of the AR; however, this interaction is only stabilised in the presence of ligand rather than ligand dependent per se [20]. Further characterisation indicated that TIP60 coactivated AR in several cell lines, including the prostate cancer cell line LNCaP and interacts with and augments activity of several other SHRs (progesterone receptor, oestrogen receptor $\alpha$ and $\beta$, and glucocorticoid receptor) $[20,21]$. Interaction with AR is dependent on an LXXLL motif present in the C-terminal domain of TIP60, mutation of which results in abolition of both interaction and coactivation [21]. Previous research had determined that TIP60 contains an HAT domain and acetylates histone proteins $\mathrm{H} 2 \mathrm{~A}, \mathrm{H} 3$, and $\mathrm{H} 4$ [22]. Using combined ${ }^{3} \mathrm{H}$-acetyl incorporation and immunoprecipitation assays, Gaughan and coworkers were able to show that TIP60, but not TIP60 HAT-domain mutants, directly acetylates AR in vivo [19]. Alanine scanning of the AR acetylation motif abolished coactivation by TIP60 in reporter gene assays providing further evidence that AR acetylation by TIP60 is an important regulatory event [19].

Conversely, proteins that can directly deacetylate AR have been reported. Histone deacetylase 1 (HDAC1) has been shown to interact directly with the AR and repress AR activity [19]. It was observed that HDAC1 interacted with a region of the AR encompassing the DBD/LBD. Furthermore, the deacetylase activity of HDAC1 was required for AR repression and although not proven, the authors suggest that direct deacetylation of AR may result in a transcriptional switch during AR-dependent gene expression [19]. In support of this, both HDAC1 and TIP60 were found to occupy the PSA promoter suggesting that the balance between acetylation and deacetylation is crucial in AR gene regulation [19].

More definitively Sirtuin 1 (SIRT1), an NAD-dependent Class III deacetylase, interacts with, deacetylates, and represses AR activity [23]. A combination of protein-protein interaction assays, use of SIRT1 inhibitors, and a catalytic "dead" SIRT1 mutant indicated that SIRT1 could regulate AR activity through direct deacetylation [23]. Interestingly, it was observed in a mammalian-two-hybrid experiment that SIRT1 abrogated p300-enhanced AR N/C-termini interactions suggesting that this event may be disrupted by deacetylation of AR hinge region [23]. Further experiments utilising an AR acetylation-deficient mutant demonstrated that this conferred resistance to SIRT1-dependent repression and, complementing previous research whereby acetylation enhanced prostate cancer cell line growth, it was found that overexpression of SIRT1 led to a decrease in cellular proliferation and colony formation. Strikingly, it was shown that SIRT1 overexpression had no discernable effect on cell lines lacking AR indicating that growth inhibition was directly linked to SIRT1-dependent AR deacetylation [23].

As outlined in Figure 1, the AR acetylation motif is situated in the flexible hinge region, which connects the structurally defined DNA-binding and ligand-binding domains [3]. Recently, it has become clear that this region impinges on a variety of steps in AR signalling including DNA binding, transactivation, and nuclear localisation [2426]. Interestingly, this region contains the NLS (amino acids
617-635), which encompasses the acetylation motif. This NLS has characteristics of a bipartite NLS such as that found in nucleoplasmin, however, it interacts with importin- $\alpha$ in a fashion that is mechanistically reminiscent of a classical or monopartite NLS [27]. Mutations identified in this region in patients with prostate cancer and androgen insensitivity syndrome (AIS) were examined and shown to confer a $\sim 10-$ 30 -fold lower affinity for importin- $\alpha$ compared to wild-type AR. When examined by confocal microscopy, the vast majority of mutant AR proteins translocated more slowly from cytoplasm to nucleus in the presence of ligand [27]. Of direct interest here is a lysine to threonine substitution at amino acid $630(\mathrm{~K} 630 \mathrm{~T})$, found in a patient with prostate cancer, which changes the AR acetylation motif KLKK to TLKK [28]. A combination of confocal microscopy, isothermal calorimetry, and reporter assays indicated that $\mathrm{AR}_{\mathrm{K} 630 \mathrm{~T}}$ had $\sim 30$-fold reduced affinity for importin- $\alpha\left(K_{\mathrm{D}}=140 \mu \mathrm{M}\right)$, migrated more slowly into the nucleus upon hormone stimulation, but paradoxically was more transcriptionally active than $\mathrm{AR}_{\mathrm{WT}}$ [27]. Independently, $\mathrm{Fu}$ and coworkers tested this mutant and found that $\mathrm{AR}_{\mathrm{K} 630 \mathrm{~T}} 0020$ was resistant to SIRT1-dependent repression [23]. These studies suggest a link between acetylation, nuclear localisation, and prostate cancer, but specific experiments to test this have not yet been reported.

Recently, the concept of interdependent transcription factor modifications has become widespread, particularly in the context of p53 signalling [29], raising the question of interdependency of acetylation and other posttranslational modifications in AR signalling. On this topic, Pestell and colleagues investigated the role of several kinases (AKT, MAPKK, PKA) in signalling through AR wild-type and AR acetylation mutants. It was observed that MAPKK signalling through AR mutant proteins was unaltered, but these mutants did affect both cAMP and AKT signalling [30]. In support of this, inhibition of cAMP signalling led to an increase of wild-type AR, but not an AR acetylation mutant, on the PSA promoter. In addition, well-characterised AR phosphorylation sites were targeted by site-directed mutagenesis to investigate any effect these phosphomutants may have on AR acetylation. Indeed, one phosphorylation site mutant (S94A) showed a decrease in transactivation of a reporter gene in response to the HDAC inhibitor trichostatin A (TSA) relative to wild-type AR [30]. Furthermore, of the three main AR phosphoisoforms, acetylation mutants lacked the hyperphosphorylated $114 \mathrm{kDa}$ form, supporting the hypothesis that acetylation and phosphorylation of $\mathrm{AR}$ are interdependent events [30]. Additional experiments must be performed to further characterise the interdependency of AR posttranslational modifications and the functional significance to signalling and gene expression.

\section{Androgen Receptor Cofactors with Histone-Acetylation Modifiying Activity}

Exhaustive lists of AR coactivators, corepressors, and coregulators have been published previously $[6,13,14,31]$. This section will focus on cofactors with histone-acetylation 
modifying activities that have been implicated in AR transcriptional regulation.

The application of chromatin immunoprecipitation (ChIP) to the study of protein-DNA interactions, epigenetics, and transcriptional regulation has vastly increased our knowledge of these processes. Indeed, recent advances in ChIP-chip and ChIP-seq technologies have enabled genomewide mapping of binding sites for nuclear receptors, including $\mathrm{AR}[32,33]$. Coregulators with HAT/HDAC activity that have been characterised by ChIP to regulate ARregulated transcription include $\mathrm{p} 300 / \mathrm{CBP}$, the p160/SRC family, P/CAF, TIP60, and HDAC1 [13].

The best-characterised AR coactivators are p300/CBP and the p160/SRC proteins. These proteins augment the transcriptional activity of multiple NRs and have been shown to be overexpressed in prostate cancer [34-36]. Steroid Receptor Coactivator-1 (SRC1) was identified as an SHR coactivator by the O'Malley laboratory, and sequence and domain interrogation studies led to the discovery that SRC1 contains an HAT domain and that this domain is integral to SHR coactivation $[37,38]$. In addition, p160 proteins were further characterised as containing specific sites for nuclear receptor interactions, a CBP interaction domain, and individual activation domains [39]. Further experimentation revealed that SRC1 could potentiate AR activity via separate interactions with $\mathrm{AR} \mathrm{N}$ - and C-termini suggesting that SRC1 binding is an integral step in full transcriptional activation of AR, possibly by recruiting further coactivators such as p300/CBP [40-42]. CBP and the highly similar p300 were initially characterised as proteins with HAT activity and thus able to regulate transcription [43-45]. Similar to p160/SRC proteins, these factors contain a HAT domain, NR binding sites but additionally sites important for interactions with E1A and CREB proteins [44]. Initial characterisation studies also proposed that these factors could recruit or bind to p160/SRC proteins thus amplifying the transcriptional response [42, 44, 46, 47].

More recently, ChIP-based investigations have reinforced the idea that p160/SRC and p300/CBP factors are central to $A R$ transcriptional regulation. Investigating the response of the AR-regulated gene encoding prostate-specific antigen (PSA) to androgen, the Brown laboratory confirmed AR binding to three AREs present in the PSA promoter; two proximal to the transcriptional start site and one ARE present in an enhancer region $\sim 4 \mathrm{~kb}$ upstream [48]. In addition to AR binding, CBP and SRC2 bound to both enhancer and promoter regions in the presence of hormone, in contrast to HDAC1 and HDAC2 and corepressors SMRT and NCoR which were able to bind PSA proximal promoter regions only in the presence of the antiandrogen bicalutamide [48]. Quantitative analysis by the laboratories of Jänne and Palvimo confirmed that SRC2 and p300 could interact with these regions and provided evidence that AR could "load" on enhancer elements to a much higher degree than at proximal promoters. Furthermore, it was observed that different loading kinetics were present, both at enhancer and promoter and between factors, implying another level of complexity in gene regulation $[49,50]$. Additional studies confirmed the presence of multiple coactivators at gene enhancers and promoters, and ChIP-3C established that chromosomal "looping" between these regions could occur, functionally linking distinct enhancer and promoter complexes $[48,51$, 52].

TIP60, discussed previously as an AR factor acetyl transferase (FAT), has more recently been found to cycle on and off the PSA promoter and enhancer regions, suggesting that this protein may regulate AR-dependent transcription at the chromatin level either through direct acetylation of AR or possibly through acetylation of chromatin [53]. Interestingly, by applying ChIP and re-ChIP techniques the authors observed that the histone deacetylase HDAC1 and the ubiquitin ligase MDM2 (murine double-minute 2) occupied the PSA promoter upon treatment with androgen, suggesting these factors may bind simulatneously or even as a complex. Furthermore, MDM2 and HDAC1 were found to co-operatively attenuate AR-regulated transcription linking ubiquitylation, acetylation and deacetylation processes. i.e. (murine double-minute 2) should be after the first reference to the protein [53].

Work from our own laboratory has indicated that proteins belonging to the same family of co-repressors may repress AR-target genes by different mechanisms. We have shown that Hairy/enhancer of split with YRPW-like motif (HEY) proteins repress AR-dependent signalling in a variety of cell lines $[54,55]$ (and paper submitted). Initially discovered in a yeast-two-hybrid screen to interact with SRC1, HEY1 was characterised as an AR corepressor. HEY1 is sensitive to a variety of HDAC inhibitors and treatment with TSA resulted in de-repression of AR activity, suggesting HEY1 repressed AR activity through the recruitment of class I/II HDACs [55]. Similar experiments performed on HEYL, a third member of the HEY family, indicated that class I/II HDAC activity was not required for AR repression (paper submitted), hence HEY proteins may employ a variety of possible mechanisms to repress AR activity.

The AR regulates multiple and varied genes at the level of transcription thus is reliant on the recruitment of distinct coregulator complexes to fine-tune transcriptional responses. It is apparent from numerous studies employing ChIP that AR recruits proteins with both HAT and HDAC activities, such as p160/SRC, p300/CBP, and HDAC1, to achieve the desired control. The emergence of genomewide ChIP will allow the dissection and comparison of transcriptional complexes at multiple genes and specific patterns of histone modifications, which will further enhance our knowledge of important coregulators.

\section{Acetylation and Prostate Cancer}

As stated above, direct acetylation of AR in response to androgen stimulation increases AR activity, response element binding, and cellular proliferation $[17,30,56,57]$. The role of acetylation in AR activity was confirmed using acetylationmimic mutants, which were found to interact with p300 to a higher degree than wild-type AR. Additionally, a panel of coactivators, such as SRC1 and TIP60, were found to coactivate these mutant ARs more robustly than wild-type 
receptor. Furthermore, these mutants conferred a growth advantage to tumour cells in vitro and in vivo suggesting that AR acetylation may play an important role in the development and/or progression of prostate cancer [16, 17, 56]. These data have been supported by the findings that SIRT1, an NAD-dependent HDAC, can deacetylate AR and reduce cellular proliferation and colony formation [23].

Androgens drive prostate tumour growth hence therapies are directed towards reducing levels of circulating androgens and AR activation via administration of luteinisinghormone releasing hormone (LHRH) analogues and/or antiandrogens. Patients generally respond well to treatment but in the majority of cases tumours progress to the advanced, androgen-independent/hormone-refractory stage of the disease. Several mechanisms can drive progression to androgenindependent prostate cancer including amplification of $A R$, altered levels of co-factors and somatic $A R$ mutations that result in promiscuous activation of the receptor by nonandrogenic ligands and growth factor pathways. $[4,58$, 59]. Patients diagnosed with androgen-independent prostate cancer have a median survival time of 12-18 months; thus, it is important to develop new treatment strategies to combat disease transition. One new development is the reduction in levels of circulating adrenal androgens using drugs such as Ketoconazole and Abiraterone, which are 450 inhibitors targeting steroid biosynthesis pathways $[58,60]$.

Several HDAC inhibitors are currently undergoing testing in clinical trials including suberoylanilide hydroxamic acid (SAHA), LBH589 and Depsipeptide, although results suggest that these treatments have moderate effects $[58,61]$. It may seem paradoxical that HDAC inhibitors are used as prostate cancer therapies since acetylation of AR increases cellular proliferation as outlined above. However, it has been proposed that HDAC inhibitors act via HDAC6 thus acetylating HSP90, known to be central in AR folding and ligand binding, or by sensitizing cells to DNA damage $[58,62$, 63]. A limited number of HAT inhibitors are in preclinical or clinical trials, with p300/CBP and P/CAF-specific inhibitors showing the most promising effects $[64,65]$. Curcumin, a naturally occurring HAT inhibitor, has been shown to inhibit CBP-dependent acetylation of histones and nonhistone proteins and seems to have a pro-apoptotic effect on prostate cancer cells in vitro and in vivo $[64,66,67]$.

\section{Concluding Remarks}

Prostate cancer growth, in early stages, is driven by androgens, and hence AR is central to disease formation and progression. As a transcription factor, AR recruits and is regulated by multiple proteins with a variety of enzymatic functions, including proteins with the ability to modify the acetylation status of chromatin and also the AR itself. These modifications allow AR to directly and acutely regulate the hormonal response, protein-protein interactions, transcriptional complexes, and importantly cellular proliferation. These observations, together with the findings that levels of cofactors with HAT/HDAC activity are overexpressed in patients with prostate cancer, have resulted in acetylation becoming an important therapeutic target.

\section{Acknowledgments}

The authors thank members of the Androgen Signalling Laboratory and Prostate Cancer Research Group for stimulating discussion and reviewing the paper and Cancer Research UK for supporting their research.

\section{References}

[1] D. M. Parkin, F. Bray, J. Ferlay, and P. Pisani, "Global cancer statistics, 2002," CA Cancer Journal for Clinicians, vol. 55, no. 2, pp. 74-108, 2005.

[2] G. R. Cunha, A. A. Donjacour, P. S. Cooke et al., "The endocrinology and developmental biology of the prostate," Endocrine Reviews, vol. 8, no. 3, pp. 338-362, 1987.

[3] E. P. Gelmann, "Molecular biology of the androgen receptor," Journal of Clinical Oncology, vol. 20, no. 13, pp. 3001-3015, 2002.

[4] B. J. Feldman and D. Feldman, "The development of androgen-independent prostate cancer," Nature Reviews Cancer, vol. 1, no. 1, pp. 32-45, 2001.

[5] A. O. Brinkmann, L. J. Blok, P. E. De Ruiter et al., "Mechanisms of androgen receptor activation and function," Journal of Steroid Biochemistry and Molecular Biology, vol. 69, no. 1-6, pp. 307-313, 1999.

[6] D. N. Lavery and I. J. McEwan, "Structure and function of steroid receptor AF1 transactivation domains: induction of active conformations," Biochemical Journal, vol. 391, no. 3, pp. 449-464, 2005.

[7] F. Claessens and D. T. Gewirth, "DNA recognition by nuclear receptors," Essays in Biochemistry, vol. 40, pp. 59-72, 2004.

[8] V. Nahoum and W. Bourguet, "Androgen and estrogen receptors: potential of crystallography in the fight against cancer," International Journal of Biochemistry and Cell Biology, vol. 39, no. 7-8, pp. 1280-1287, 2007.

[9] P. M. Matias, P. Donner, R. Coelho et al., "Structural evidence for ligand specificity in the binding domain of the human androgen receptor: implications for pathogenic gene mutations," Journal of Biological Chemistry, vol. 275, no. 34, pp. 26164-26171, 2000.

[10] G. Jenster, H. A. G. M. Van der Korput, J. Trapman, and A. O. Brinkmann, "Identification of two transcription activation units in the N-terminal domain of the human androgen receptor," Journal of Biological Chemistry, vol. 270, no. 13, pp. 7341-7346, 1995.

[11] J. A. Simental, M. Sar, M. V. Lane, F. S. French, and E. M. Wilson, "Transcriptional activation and nuclear targeting signals of the human androgen receptor," Journal of Biological Chemistry, vol. 266, no. 1, pp. 510-518, 1991.

[12] G. Jenster, H. A. G. M. Van der Korput, C. Van Vroonhoven, T. H. Van der Kwast, J. Trapman, and A. O. Brinkmann, "Domains of the human androgen receptor involved in steroid binding, transcriptional activation, and subcellular localization," Molecular Endocrinology, vol. 5, no. 10, pp. 13961404, 1991.

[13] H. V. Heemers and D. J. Tindall, "Androgen receptor (AR) coregulators: a diversity of functions converging on and regulating the AR transcriptional complex," Endocrine Reviews, vol. 28, no. 7, pp. 778-808, 2007. 
[14] R. Chmelar, G. Buchanan, E. F. Need, W. Tilley, and N. M. Greenberg, "Androgen receptor coregulators and their involvement in the development and progression of prostate cancer," International Journal of Cancer, vol. 120, no. 4, pp. 719-733, 2007.

[15] R. D. Ward and N. L. Weigel, "Steroid receptor phosphorylation: assigning function to site-specific phosphorylation," BioFactors, vol. 35, no. 6, pp. 528-536, 2009.

[16] M. Fu, C. Wang, A. T. Reutens et al., "p300 and p300/cAMPresponse element-binding protein-associated factor acetylate the androgen receptor at sites governing hormone-dependent transactivation," Journal of Biological Chemistry, vol. 275, no. 27, pp. 20853-20860, 2000.

[17] M. Fu, M. Rao, C. Wang et al., "Acetylation of androgen receptor enhances coactivator binding and promotes prostate cancer cell growth," Molecular and Cellular Biology, vol. 23, no. 23, pp. 8563-8575, 2003.

[18] M. Fu, C. Wang, X. Zhang, and R. Pestell, "Nuclear receptor modifications and endocrine cell proliferation," Journal of Steroid Biochemistry and Molecular Biology, vol. 85, no. 2-5, pp. 133-138, 2003.

[19] L. Gaughan, I. R. Logan, S. Cook, D. E. Neal, and C. N. Robson, "Tip60 and histone deacetylase 1 regulate androgen receptor activity through changes to the acetylation status of the receptor," Journal of Biological Chemistry, vol. 277, no. 29, pp. 25904-25913, 2002.

[20] M. E. Brady, D. M. Ozanne, L. Gaughan et al., "Tip60 is a nuclear hormone receptor coactivator," Journal of Biological Chemistry, vol. 274, no. 25, pp. 17599-17604, 1999.

[21] L. Gaughan, M. E. Brady, S. Cook, D. E. Neal, and C. N. Robson, "Tip60 is a co-activator specific for class I nuclear hormone receptors," Journal of Biological Chemistry, vol. 276, no. 50, pp. 46841-46848, 2001.

[22] T. Yamamoto and M. Horikoshi, "Novel substrate specificity of the histone acetyltransferase activity of HIV-1-Tat interactive protein Tip60," Journal of Biological Chemistry, vol. 272, no. 49, pp. 30595-30598, 1997.

[23] M. Fu, M. Liu, A. A. Sauve et al., "Hormonal control of androgen receptor function through SIRT1," Molecular and Cellular Biology, vol. 26, no. 21, pp. 8122-8135, 2006.

[24] T. Tanner, F. Claessens, and A. Haelens, "The hinge region of the androgen receptor plays a role in proteasome-mediated transcriptional activation," Annals of the New York Academy of Sciences, vol. 1030, pp. 587-592, 2004.

[25] A. Haelens, T. Tanner, S. Denayer, L. Callewaert, and F. Claessens, "The hinge region regulates DNA binding, nuclear translocation, and transactivation of the androgen receptor," Cancer Research, vol. 67, no. 9, pp. 4514-4523, 2007.

[26] T. M. Tanner, S. Denayer, B. Geverts et al., "A RKLKK motif in the hinge region controls the androgen receptor at multiple levels," Cellular and Molecular Life Sciences, vol. 67, no. 11, pp. 1919-1927, 2010.

[27] M. L. Cutress, H. C. Whitaker, I. G. Mills, M. Stewart, and D. E. Neal, "Structural basis for the nuclear import of the human androgen receptor," Journal of Cell Science, vol. 121, no. 7, pp. 957-968, 2008.

[28] W. D. Tilley, G. Buchanan, T. E. Hickey, and J. M. Bentel, "Mutations in the androgen receptor gene are associated with progression of human prostate cancer to androgen independence," Clinical Cancer Research, vol. 2, no. 2, pp. 277285, 1996.

[29] D. W. Meek and C. W. Anderson, "Posttranslational modification of p53: cooperative integrators of function," Cold Spring
Harbor Perspectives in Biology, vol. 1, no. 6, Article ID a000950, 2009.

[30] M. Fu, M. Rao, K. Wu et al., "The androgen receptor acetylation site regulates cAMP and AKT but not ERKinduced activity," Journal of Biological Chemistry, vol. 279, no. 28, pp. 29436-29449, 2004.

[31] L. Wang, C. L. Hsu, and C. Chang, "Androgen receptor corepressors: an overview," Prostate, vol. 63, no. 2, pp. 117130, 2005.

[32] L. I. Jia, B. P. Berman, U. Jariwala et al., "Genomic androgen receptor-occupied regions with different functions, defined by histone acetylation, coregulators and transcriptional capacity," PLoS ONE, vol. 3, no. 11, Article ID e3645, 2008.

[33] M. Kininis and W. L. Kraus, "A global view of transcriptional regulation by nuclear receptors: gene expression, factor localization, and DNA sequence analysis," Nuclear Receptor Signaling, vol. 6, aticle e005, 2008.

[34] J. Xu, R. C. Wu, and B. W. O'Malley, "Normal and cancerrelated functions of the p160 steroid receptor co-activator (SRC) family," Nature Reviews Cancer, vol. 9, no. 9, pp. 615630, 2009.

[35] N. G. Iyer, H. Özdag, and C. Caldas, "p300/CBP and cancer," Oncogene, vol. 23, no. 24, pp. 4225-4231, 2004.

[36] Z. Culig, B. Comuzzi, H. Steiner, G. Bartsch, and A. Hobisch, "Expression and function of androgen receptor coactivators in prostate cancer," Journal of Steroid Biochemistry and Molecular Biology, vol. 92, no. 4, pp. 265-271, 2004.

[37] T. E. Spencer, G. Jenster, M. M. Burcin et al., "Steroid receptor coactivator-1 is a histone acetyltransferase," Nature, vol. 389, no. 6647, pp. 194-198, 1997.

[38] S. A. Onate, V. Boonyaratanakornkit, T. E. Spencer et al., "The steroid receptor coactivator-1 contains multiple receptor interacting and activation domains that cooperatively enhance the activation function 1 (AF1) and AF2 domains of steroid receptors," Journal of Biological Chemistry, vol. 273, no. 20, pp. 12101-12108, 1998.

[39] N. J. McKenna, R. B. Lanz, and B. W. O’Malley, "Nuclear receptor coregulators: cellular and molecular biology," Endocrine Reviews, vol. 20, no. 3, pp. 321-344, 1999.

[40] C. L. Bevan, S. Hoare, F. Claessens, D. M. Heery, and M. G. Parker, "The AF1 and AF2 domains of the androgen receptor interact with distinct regions of SRC1," Molecular and Cellular Biology, vol. 19, no. 12, pp. 8383-8392, 1999.

[41] T. Ikonen, J. J. Palvimo, and O. A. Jänne, "Interaction between the amino- and carboxyl-terminal regions of the rat androgen receptor modulates transcriptional activity and is influenced by nuclear receptor coactivators," Journal of Biological Chemistry, vol. 272, no. 47, pp. 29821-29828, 1997.

[42] C. L. Smith, S. A. Oñate, M. J. Tsai, and B. W. O’Malley, "CREB binding protein acts synergistically with steroid receptor coactivator-1 to enhance steroid receptor-dependent transcription," Proceedings of the National Academy of Sciences of the United States of America, vol. 93, no. 17, pp. 8884-8888, 1996.

[43] A. J. Bannister and T. Kouzarides, "The CBP co-activator is a histone acetyltransferase," Nature, vol. 384, no. 6610, pp. 641643, 1996.

[44] N. Vo and R. H. Goodman, "CREB-binding Protein and p300 in Transcriptional Regulation," Journal of Biological Chemistry, vol. 276, no. 17, pp. 13505-13508, 2001.

[45] V. V. Ogryzko, R. L. Schiltz, V. Russanova, B. H. Howard, and Y. Nakatani, "The transcriptional coactivators p300 and CBP are histone acetyltransferases," Cell, vol. 87, no. 5, pp. 953-959, 1996. 
[46] T. P. Yao, G. Ku, N. Zhou, R. Scully, and D. M. Livingston, "The nuclear hormone receptor coactivator SRC-1 is a specific target of p300," Proceedings of the National Academy of Sciences of the United States of America, vol. 93, no. 20, pp. 1062610631, 1996.

[47] N. J. Mckenna, Z. Nawaz, S. Y. Tsai, M. J. Tsai, and B. W. O’Malley, "Distinct steady-state nuclear receptor coregulator complexes exist in vivo," Proceedings of the National Academy of Sciences of the United States of America, vol. 95, no. 20, pp. 11697-11702, 1998.

[48] Y. Shang, M. Myers, and M. Brown, "Formation of the androgen receptor transcription complex," Molecular Cell, vol. 9, no. 3, pp. 601-610, 2002.

[49] Z. Kang, O. A. Jänne, and J. J. Palvimo, "Coregulator recruitment and histone modifications in transcriptional regulation by the androgen receptor," Molecular Endocrinology, vol. 18, no. 11, pp. 2633-2648, 2004.

[50] Z. Kang, A. Pirskanen, O. A. Jänne, and J. J. Palvimo, "Involvement of proteasome in the dynamic assembly of the androgen receptor transcription complex," Journal of Biological Chemistry, vol. 277, no. 50, pp. 48366-48371, 2002.

[51] Q. Wang, J. S. Carroll, and M. Brown, "Spatial and temporal recruitment of androgen receptor and its coactivators involves chromosomal looping and polymerase tracking," Molecular Cell, vol. 19, no. 5, pp. 631-642, 2005.

[52] M. C. Louie, H. Q. Yang, AI. H. Ma et al., "Androgen-induced recruitment of RNA polymerase II to a nuclear receptor-p160 coactivator complex," Proceedings of the National Academy of Sciences of the United States of America, vol. 100, no. 5, pp. 2226-2230, 2003.

[53] L. Gaughan, I. R. Logan, D. E. Neal, and C. N. Robson, "Regulation of androgen receptor and histone deacetylase 1 by Mdm2-mediated ubiquitylation," Nucleic Acids Research, vol. 33, no. 1, pp. 13-26, 2005.

[54] M. A. Villaronga, D. N. Lavery, C. L. Bevan, S. Llanos, and B. Belandia, "HEY1 Leu94Met gene polymorphism dramatically modifies its biological functions," Oncogene, vol. 29, no. 3, pp. 411-420, 2010.

[55] B. Belandia, S. M. Powell, J. M. García-Pedrero, M. M. Walker, C. L. Bevan, and M. G. Parker, "Hey1, a mediator of Notch signaling, is an androgen receptor corepressor," Molecular and Cellular Biology, vol. 25, no. 4, pp. 1425-1436, 2005.

[56] M. Fu, C. Wang, X. Zhang, and R. G. Pestell, "Acetylation of nuclear receptors in cellular growth and apoptosis," Biochemical Pharmacology, vol. 68, no. 6, pp. 1199-1208, 2004.

[57] V. M. Popov, C. Wang, L. A. Shirley et al., "The functional significance of nuclear receptor acetylation," Steroids, vol. 72, no. 2, pp. 221-230, 2007.

[58] YU. Chen, C. L. Sawyers, and H. I. Scher, "Targeting the androgen receptor pathway in prostate cancer," Current Opinion in Pharmacology, vol. 8, no. 4, pp. 440-448, 2008.

[59] G. N. Brooke and C. L. Bevan, "The role of androgen receptor mutations in prostate cancer progression," Current Genomics, vol. 10, no. 1, pp. 18-25, 2009.

[60] G. Attard, D. Sarker, A. Reid, R. Molife, C. Parker, and J. S. De Bono, "Improving the outcome of patients with castrationresistant prostate cancer through rational drug development," British Journal of Cancer, vol. 95, no. 7, pp. 767-774, 2006.

[61] LI. Shen and R. Pili, "Posttranscription regulation of prostate cancer growth," Cancer Journal, vol. 14, no. 1, pp. 46-53, 2008.

[62] L. Chen, S. Meng, H. Wang et al., "Chemical ablation of androgen receptor in prostate cancer cells by the histone deacetylase inhibitor LAQ824," Molecular Cancer Therapeutics, vol. 4, no. 9, pp. 1311-1319, 2005.
[63] C. S. Chen, YU. C. Wang, H. C. Yang et al., "Histone deacetylase inhibitors sensitize prostate cancer cells to agents that produce DNA double-strand breaks by targeting Ku70 acetylation," Cancer Research, vol. 67, no. 11, pp. 5318-5327, 2007.

[64] F. Manzo, F. P. Tambaro, A. Mai, and L. Altucci, "Histone acetyltransferase inhibitors and preclinical studies," Expert Opinion on Therapeutic Patents, vol. 19, no. 6, pp. 761-774, 2009.

[65] F. J. Dekker and H. J. Haisma, "Histone acetyl transferases as emerging drug targets," Drug Discovery Today, vol. 14, no. 1920, pp. 942-948, 2009.

[66] K. Balasubramanyam, R. A. Varier, M. Altaf et al., "Curcumin, a novel p300/CREB-binding protein-specific inhibitor of acetyltransferase, represses the acetylation of histone/nonhistone proteins and histone acetyltransferasedependent chromatin transcription," Journal of Biological Chemistry, vol. 279, no. 49, pp. 51163-51171, 2004.

[67] N. Khan, V. M. Adhami, and H. Mukhtar, "Apoptosis by dietary agents for prevention and treatment of prostate cancer," Endocrine-Related Cancer, vol. 17, no. 1, pp. R39-R52, 2010. 

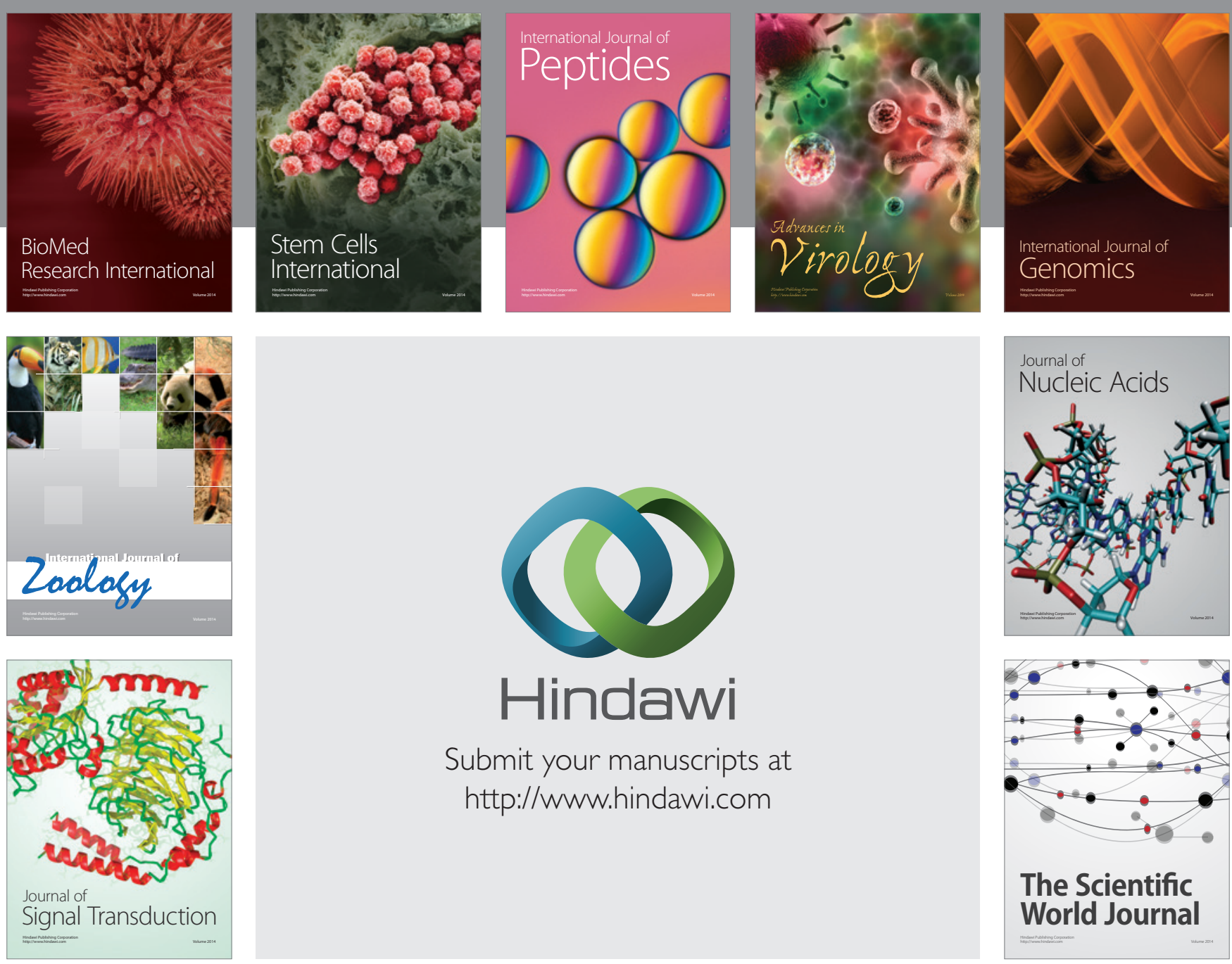

Submit your manuscripts at

http://www.hindawi.com
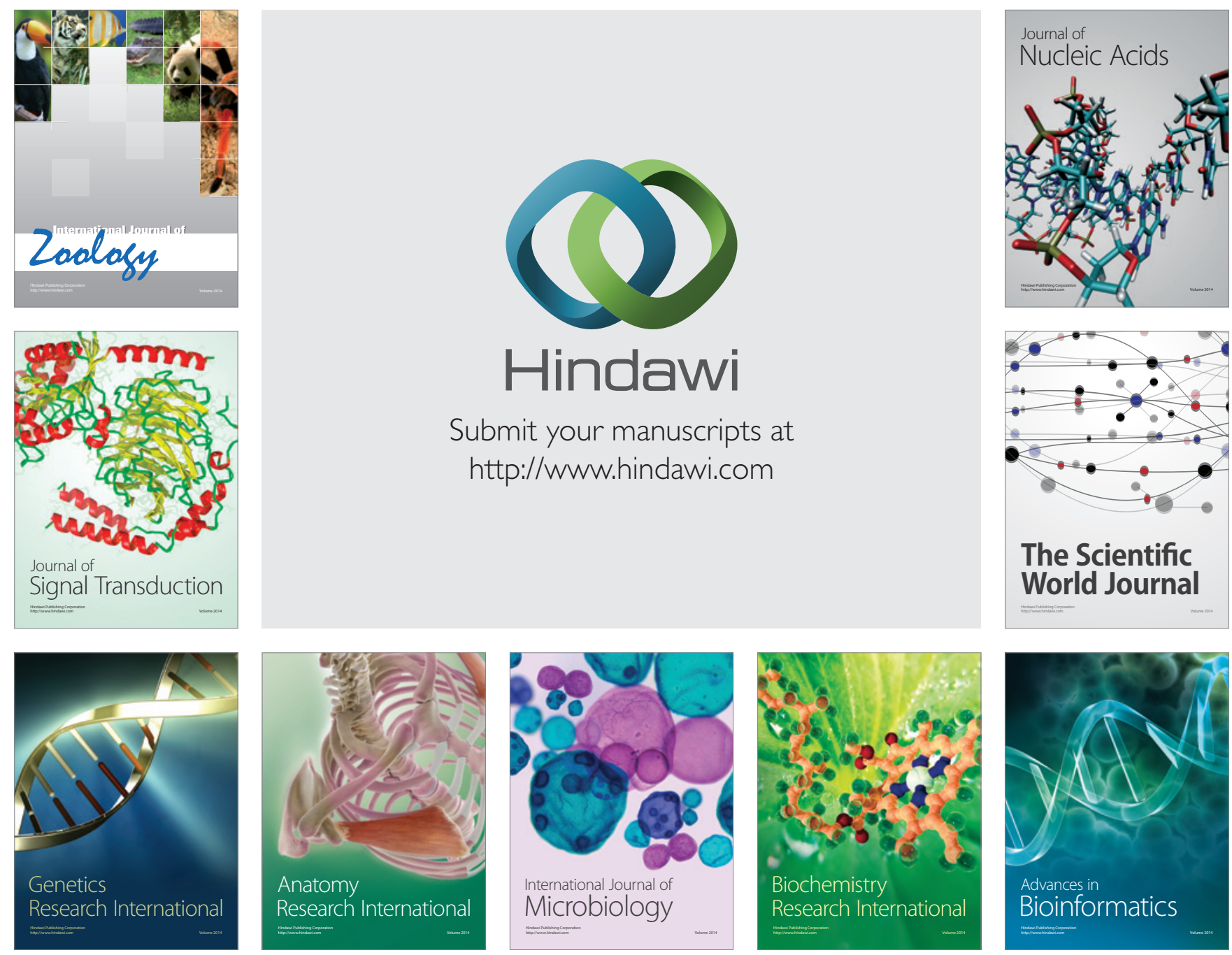

The Scientific World Journal
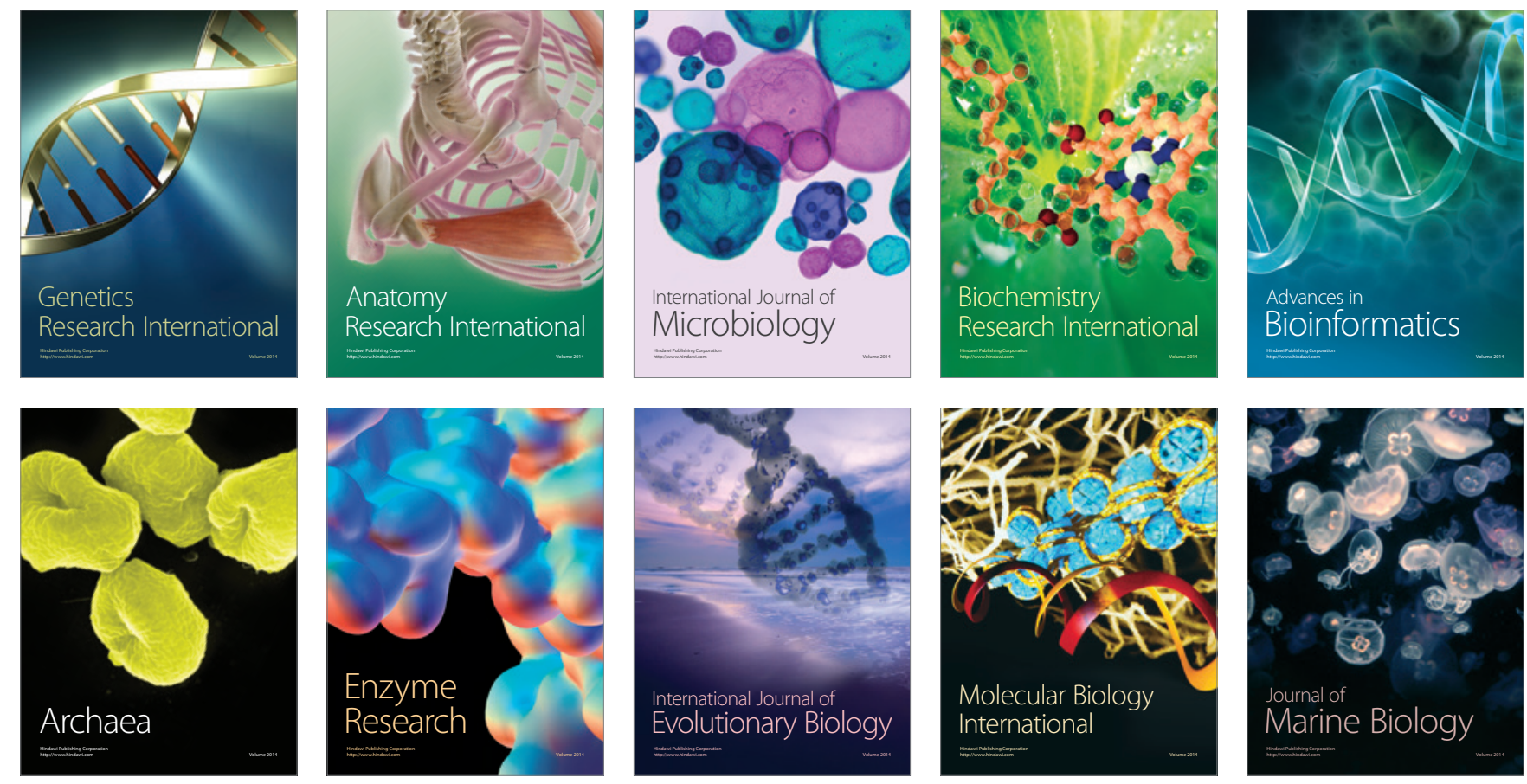